\title{
Selección y Diseño de una Máquina extractora de aceite del piñón Jatropha curcas
}

\author{
Selection and Design of a Jatropha curcas pinion oil extractor machine
}

Paco Jovanni Vásquez Carrera. ${ }^{1}$, William Armando Hidalgo Osorio. ${ }^{2}$, Guido Gabriel Carrillo Velarde. ${ }^{3} \&$ Nelson Jonathan Villarroel Herrera. ${ }^{4}$

Recibido: 05-07-2020 / Revisado: 07-08-2020 / Aceptado: 08-09-2020 / Publicado: 03-10-2020

\section{Resumen.}

En la actualidad es de gran importancia dar a conocer diferentes alternativas que se puede usar para bajar el gran índice de contaminación al que estamos expuestos por el uso de derivados del petróleo en la aplicación de fluidos de corte por ende como se plantea el diseño de una máquina, que permita obtener un fluido de corte de mediante el aceite de piñón (Jatropha Curcas).

El objetivo se direccionó a seleccionar y diseñar una máquina de extracción de aceite de piñón. Los métodos aplicados para la elaboración de este artículo fue la revisión documental - bibliográfica de varios autores, y analítico. Posterior a la revisión bibliográfica se identificó que existen variedad de máquinas de expeller de simple y doble tornillo. Entre los cuales se seleccionó la máquina expeller de un solo tornillo, y se procedió al diseño de la misma llegando a la conclusión que para el buen funcionamiento de la misma se necesita construir el tornillo de acero AISI 304, para que resista la corrosión y fatiga producto de la presión ejercida entre el material el tornillo y la camisa. Los cilindros tienen que fabricarse de acero AISI 4140 y 4340; para que sean resistentes a la fatiga y fractura y para su operación un motorreductor de $6 \mathrm{Hp}$, el mismo que producirá un torque de $412 \mathrm{Nm}$, un variador de velocidad ACS355-03E-17A6-2 y termocuplas tipo J 3/8 x 12 in. Con este diseño se tendrá una producción de $30 \mathrm{Kg} / \mathrm{h}$.

\footnotetext{
${ }^{1}$ Universidad Técnica de Cotopaxi, La Maná, Ecuador, paco.vasquez@utc.edu.ec

${ }^{2}$ Universidad Técnica de Cotopaxi, La Maná, Ecuador, william.hidalgo7885@utc.edu.ec

${ }^{3}$ Universidad Técnica de Cotopaxi, La Maná, Ecuador, guido.carrillo2303@utc.edu.ec

${ }^{4}$ Universidad Técnica de Cotopaxi, La Maná, Ecuador, nelson.villarroel3254@utc.edu.ec
} 
Palabras claves: Tornillo expeller, motorreductor, termocupla tipo J, variador de velocidad, aceite de piñón.

\begin{abstract}
.
At present it is of great importance to publicize different alternatives that can be used to lower the large pollution index to which we are exposed by the use of petroleum derivatives in the application of cutting fluids, therefore, as the design of a machine, which allows to obtain a cutting fluid from using pinion oil (Jatropha Curcas).

The objective was to select and design a pinion oil extraction machine. The methods applied for the elaboration of this article was the documentary - bibliographic review of several authors, and analytical. After the literature review, it was identified that there are a variety of single and double screw expeller machines. Among which the single screw expeller machine was selected, and it was designed to conclude that it is necessary to build the AISI 304 steel screw for its proper operation, so that it resists corrosion and fatigue product of the pressure exerted between the material the screw and the sleeve. The cylinders have to be made of AISI 4140 and 4340 steel; so that they are resistant to fatigue and fracture and for their operation a $6 \mathrm{Hp}$ gearmotor, the same that will produce a torque of $412 \mathrm{Nm}$, a speed variator ACS355-03E-17A6-2 and thermocouples type J 3/8 x 12 in. With this design there will be a production of $30 \mathrm{Kg} / \mathrm{h}$.
\end{abstract}

Keywords: Expeller screw, gearmotor, J type thermocouple, speed variator, pinion oil.

\title{
Introducción.
}

En los últimos se ha venido haciendo cada vez más necesaria la obtención de energías renovables a fin de poder combatir con alternativas concretas el cambio climático.

Los aceites vegetales presentan una valiosa clase de tales recursos renovables. En la industria, la extracción de aceite más comúnmente aplica proceso para semillas con un contenido de aceite relativamente alto, es decir $20 \%$, comprende tanto un paso de prensado, como como una extracción con solver. Tradicionalmente el prensado se realiza mediante el uso de una prensa hidráulica, pero desde medianos del siglo XX, se utiliza un sistema de extracción por tornillo para el prensado mecánico de semillas oleaginosas. (Uitterhaegen \& Evon, 2017)

La máquina expeller es esencialmente una prensa de jaula, en la que la presión se desarrolla por medio de un eje rotatorio o tornillo sinfín en vez de un pistón de acción intermitente. En la jaula se desarrolla una presión extremadamente alta, del orden de 140 a $2800 \mathrm{~kg} / \mathrm{cm} 2$, por medio de la acción del eje contra un orificio o estrangulación regulable, que restringe la descarga de la torta al extremo de la jaula.

La necesidad de contar con esta clase de máquinas en la Industria, ya que presenta un buen rendimiento de volumen de aceite extraído por ser un proceso continuo y tener una capacidad alta de producción. 
Los aspectos antes mencionados muestran la necesidad de desarrollar una máquina de extracción del aceite del piñón, cuyo costo sea accesible a comparación con las opciones disponibles en el mercado.

El objetivo del presente artículo es el diseño de una máquina extractora de aceite del piñón Jatropha curcas, que aparte de obtener la materia prima para un fluido de corte dotara al estudiante de una herramienta de trabajo para realizar prácticas en las asignaturas de protecciones eléctricas, control industrial, procesos de manufactura, instrumentación, entre otras.

Se han realizado diversos trabajos investigativos, con el objetivo de extraer aceites vegetales:

Extracción de aceite de semillas de Jatropha mediante un extrusor de doble tornillo, en el que el rendimiento de extracción de aceite aumentó al disminuir la temperatura y la velocidad de rotación del tornillo. El mayor rendimiento de extracción de aceite (70.6\%) con una buena calidad de la torta de prensa (contenido de aceite residual inferior al 8\%) se obtuvo bajo condiciones de operación de $153 \mathrm{rpm}$ de velocidad de rotación del tornillo, $5.16 \mathrm{~kg} / \mathrm{h}$ de flujo de entrada de semillas de Jatropha, y presión a $80^{\circ} \mathrm{C}$ temperatura. El aceite expresado correspondiente fue barato de producir $(71 \mathrm{~W} \mathrm{~h} / \mathrm{kg}$ de semilla procesada o $314 \mathrm{~W} \mathrm{~h} / \mathrm{kg}$ de aceite expresado para energía mecánica específica) en comparación con otra técnica continua, es decir, la prensa de un solo expulsor, comúnmente utilizada para la extracción mecánica de aceite de Jatropha. (Evon, Kartika, \& Rigal, 2013)

En el 2017, en Touluse - Francia, en la Universidad de Touluse, se realizó una Tecnología de extrusión de doble tornillo para la extracción de aceite vegetal. Este trabajo se enfocó en la extrusión de tornillo para extracción de aceite vegetal y revisa los últimos avances tecnológicos. (Uitterhaegen \& Evon, 2017)

En el 2019, en Akoka, - Nigeria, en la Universidad de Lagos, se llevó acabo el análisis paramétrico y diseño de extrusora de tornillo recto para compactación de sólidos, mediante el análisis del efecto de la velocidad del tornillo en el caudal de arrastre. (Orisaleye \& Ojolo, 2019)

En el 2019, el Uso energético de los desechos de extracción de aceite de Jatropha: pellets de biochar y mezclas de cáscara de Jatropha; permitió determinar la combustión de pellets hechos con recursos renovables en un quemador industrial mostro temperaturas promedio que oscilan entre 300 y $350{ }^{\circ} \mathrm{C}$, con una temperatura máxima de $460{ }^{\circ} \mathrm{C}$.

El costo de producción del pellet seleccionado se estima en 0,11 USD/kg, lo que hace que el costo de energía sea aproximadamente el 28\% del costo de energía del GLP. (Ramiréz, Martí, \& Rivadeneira, 2019)

\section{Extrusora de tornillo.}

Es una máquina que su funcionamiento se basa en un tornillo extrusor y una jaula, en la que el tornillo transporta el material, y su capacidad volumétrica depende del diámetro y la longitud del mismo. 
Figura 1. Extrusora de tornillo.

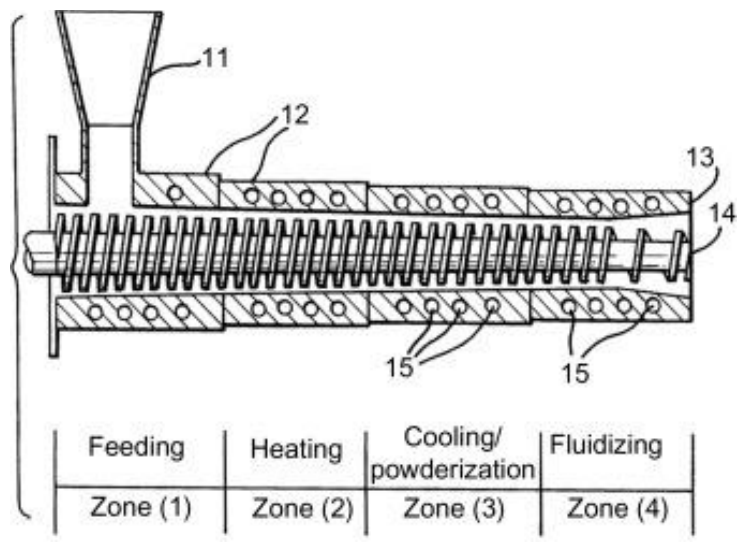

Fuente: (Ali, 2016)

\section{Expeller de Tornillo simple.}

El expeller de tornillo simple mostrado en la figura 2, consiste en un solo husillo que gira libremente dentro de un cilindro, barril o camisa; estos dos elementos interactúan a manera de una bomba elevando la presión de la mezcla, extrayendo el aceite y drenándolo a través de las ranuras u orificios y liberando la torta por medio de un agujero llamado dado.

Para elevar la presión en un tornillo simple existen variaciones de geometría que influyen directamente en el comportamiento de la mezcla mientras permanece dentro del cilindro, además la forma del tornillo también influye en la potencia requerida para la extrusión y por ende en el calor generado en el proceso, dado que gran parte de la energía se libera en calor.

Figura 2. Extrusora de tornillo.

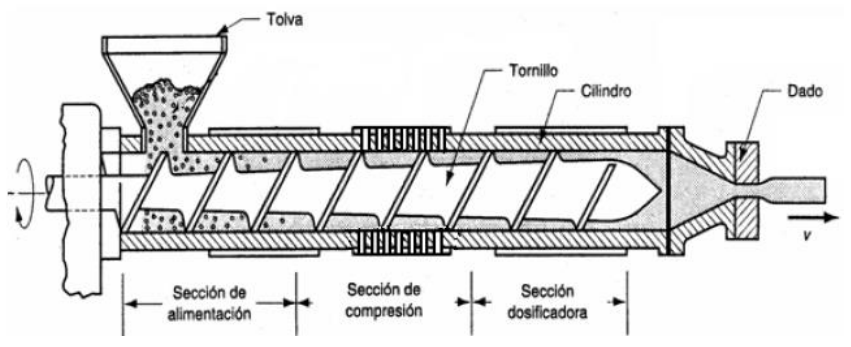

Fuente: (Gómez Vargas \& Villegas Zapater, 2011)

En la jaula se desarrolla una presión extremadamente alta, del orden de 140 a $2800 \mathrm{~kg} / \mathrm{cm} 2$, por medio de la acción del eje contra un orificio o estrangulación regulable, que restringe la descarga de la torta al extremo de la jaula.

\section{Metodología.}


El presente proyecto se enmarcó en la investigación documental - bibliográfico, enfocada en la selección y diseño del tipo de máquina expeller. En primera instancia se determinó el tipo de mecanismo de la máquina para extracción del aceite, optando por la extrusión por un solo tornillo, así como la elección del variador de velocidad y las resistencias para el funcionamiento adecuado de la máquina.

Se seleccionó las partes constitutivas de la máquina extrusora de tornillo como mecánicas: tornillo extrusor, rodamientos cónicos, camisas en zonas de alimentación y proceso, tolva y bastidor para el motorreductor y los elementos eléctricos y electrónicos: Variador de velocidad, Fusibles, pulsadores on/off, focos indicadores de encendido y falla, motor eléctrico y el sistema de calentamiento del expeller con resistencias tipo J y controlador ATR2415. Después de la selección de los diferentes componentes, se procedió al diseño del sistema mecánico de la máquina en Inventor.

\section{Resultados y discusión.}

\section{Matriz de selección de la alternativa de la máquina de extracción de aceite.}

Para la elección de la máquina para la extracción del piñón de aceite se consideró los siguientes parámetros.

Costos de fabricación. - Al tener dos tornillos se cuadriplica el costo de la máquina por la construcción de los dos tornillos y por la forma particular de la camisa que tiene que adaptarse a los dos tornillos.

El costo de una prensa de un solo tornillo GX-85 es de 500 y de doble tornillo GN 86 es de 2000 USD, pero en Estados Unidos.

(Global trade starts here, 2019)

Factibilidad de diseño. - El expeller de doble tornillo presenta un diseño bastante complejo, puesto que en el diseño de estos tornillos la geometría variable no es posible dado que los dos tornillos deben ser solidarios entre sí.

Capacidad de Producción. - Existen en el mercado máquinas de expeller simple Modelos GX con capacidad desde 70 a $400 \mathrm{Kg} / \mathrm{h}$., y las máquinas de doble tornillo en modelos GN con capacidad que varía desde 110 a $500 \mathrm{~kg} / \mathrm{h}$. (Global trade starts here, 2019)

Factibilidad tecnológica de construcción. - En este aspecto particular la tecnología presente en el medio, es más factible para la construcción de un solo expeller que el de doble expeller, por presentar restricciones como un sistema motriz único de ambos ejes.

Facilidad de operación. - Tanto la máquina de un solo tornillo como de dos tornillos presentan la misma facilidad de operación y funcionamiento. El principio de funcionamiento es similar en los dos sistemas, puesto que tienen zona de alimentación, compresión y dosificación. 
Mantenimiento. - Los expeller de doble tornillo tienen que lubricarse el mecanismo motriz de ambos ejes, a diferencia del expeller de un solo tornillo q va acoplado directamente al motorreductror.

Eficiencia mecánica. - Un tronillo de doble husillo permite una mejor mezcla de material y una mejor eficiencia de la energía mecánica de entrada.

En la tabla 1, se muestra la ponderación para la elección de la máquina en base a todos los parámetros descritos anteriormente.

Tabla 1. Matriz de selección de la alternativa de la máquina de extracción de aceite.

\begin{tabular}{lccc}
\hline \multicolumn{1}{c}{ Parámetros } & $\begin{array}{c}\text { Calificación en } \\
\text { importancia/100 }\end{array}$ & $\begin{array}{c}\text { Expeller } \\
\text { tornillo simple }\end{array}$ & $\begin{array}{c}\text { Expeller tornillo } \\
\text { doble }\end{array}$ \\
\hline $\begin{array}{l}\text { Costos de fabricación } \\
\begin{array}{l}\text { Factibilidad de } \\
\text { diseño }\end{array}\end{array}$ & 25 & 25 & 15 \\
$\begin{array}{l}\text { Capacidad de } \\
\text { producción }\end{array}$ & 20 & 20 & 15 \\
$\begin{array}{l}\text { Facilidad tecnológica } \\
\text { de construcción }\end{array}$ & 15 & 10 & 15 \\
$\begin{array}{l}\text { Facilidad de } \\
\text { operación }\end{array}$ & 10 & 10 & 7 \\
$\begin{array}{l}\text { Mantenimiento } \\
\text { Eficiencia mecánica }\end{array}$ & 10 & 10 & 10 \\
$\quad$ Resultado & 10 & 10 & 6 \\
\hline
\end{tabular}

Fuente: (Gómez Vargas \& Villegas Zapater, 2011)

\section{Parámetros de operación del producto}

Temperatura. - Para poder obtener la mejor eficiencia mecánica la semilla de piñón debe encontrarse en un rango de temperatura que no supere $\operatorname{los} 90^{\circ} \mathrm{C}$ según, experimentos realizados en la ESPE, para que el fluido no se disipe en calor. Pero en la zona de dosificación la temperatura debe ser mayor a $90^{\circ} \mathrm{C}$ para que el producto a extruirse tenga facilidad de desfogue.

Humedad. - Investigaciones realizadas por parte de la Escuela Politécnica Nacional, han determinado que la humedad óptima de almacenamiento y previa a la extracción de aceite, oscila entre el 7\%,8\% y hasta 12\% de humedad. (Gómez Vargas \& Villegas Zapater, 2011)

\section{Cálculo del torque y potencia del motorreductor}

Acorde a métodos experimentales realizados por la ESPE y EPN, se obtiene que para la extracción del aceite del piñón se requiere una presión de $30 \mathrm{MPa}$, mediante esta presión se realizó los cálculos 
para determinar el torque del motorreductor. En la tabla 2, se muestra los resultados de los cálculos realizados.

Tabla 2. Cálculo del torque en el tornillo

\begin{tabular}{lcc}
\hline \multicolumn{1}{c}{ VARIABLES } & \multicolumn{2}{c}{ UNIDADES } \\
\hline Presión experimental & {$[\mathrm{kg} / \mathrm{cm} 2]$} & {$[\mathrm{MPa}]$} \\
& 305,91 & 30 \\
& {$[\mathrm{~cm} 2]$} & {$[\mathrm{m} 2]$} \\
Área del piñon & 1,40 & 0,00014022 \\
& {$[\mathrm{kgf}]$} & {$[\mathrm{N}]$} \\
Fuerza & 428,95 & 4203,68 \\
& & \\
Número de pepas & 100 & \\
& & \\
Fuerza total & 42894,70 & {$[\mathrm{~N}]$} \\
Radio de separación & 0,006 & {$[\mathrm{~m}]$} \\
Torque requerido & $\mathbf{2 5 7 , 3 7}$ & {$[\mathbf{N m}]$} \\
\hline
\end{tabular}

Fuente: Elaboración propia.

Con el torque establecido se puede determinar en base al catálogo técnico Motores ortogonales Standard IEC, el motorreductor, siendo seleccionado acorde al torque de $261 \mathrm{Nm}$, que es el que se dispone en el mercado:

Motor TS112MB4 y el reductor BO83 mostrado en la figura 3, con un factor de servicio de 2. El factor de servicio se determinó del producto del coeficiente de las 16 horas de trabajo diarias $\mathrm{Cd}=1,5$ por el coeficiente del número de arranques $\mathrm{Cf}=1,3$.

Figura 3. Motor y reductor en la marca MOTOVARIO

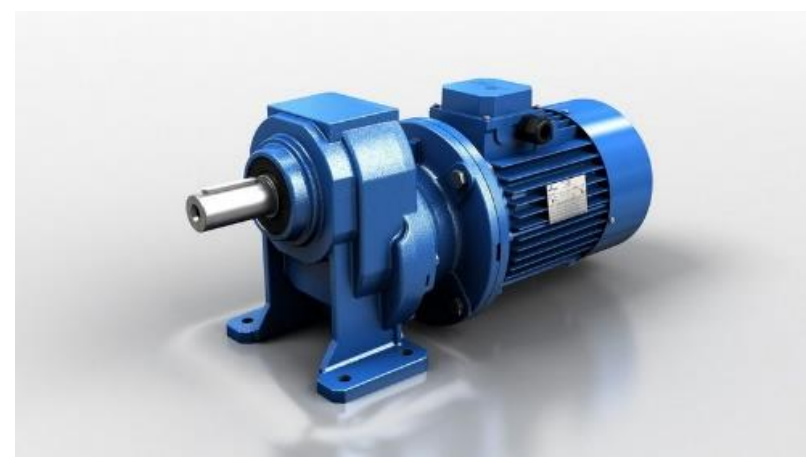

Fuente: Elaboración propia. 
En la tabla 3, se muestra las características del motorreductor.

Tabla 3. Datos del motorreductor

\begin{tabular}{lcc}
\hline \multicolumn{1}{c}{ VARIABLES } & VALOR & UNIDADES \\
\hline $\begin{array}{l}\text { Potencia motor } \\
\text { eléctrico }\end{array}$ & 4,8 & {$[\mathrm{~kW}]$} \\
$\begin{array}{l}\text { Revoluciones por } \\
\text { minuto }\end{array}$ & 1740 & {$[\mathrm{rpm}]$} \\
$\begin{array}{l}\text { Potencia a la salida } \\
\text { del motor }\end{array}$ & 4,8 & {$[\mathrm{~kW}]$} \\
$\begin{array}{l}\text { Velocidad de salida } \\
\text { del motoreductor }\end{array}$ & 105 & {$[\mathrm{rpm}]$} \\
$\begin{array}{l}\text { Par requerido en el } \\
\text { árbol lento }\end{array}$ & 400 & {$[\mathrm{Nm}]$} \\
\hline
\end{tabular}

Fuente: (MOTOVARIO, 2018)

Como resultado de la tabla 4, tenemos que el aumento de la capacidad de producción es directamente proporcional al aumento de la potencia e inversamente proporcional a las revoluciones por minuto.

Tabla 4. Comparación de máquinas de extracción.

\begin{tabular}{|c|c|c|c|c|c|c|c|}
\hline \multicolumn{8}{|c|}{ MÁQUINAS CON UN SOLO EXPELLER } \\
\hline \multirow{4}{*}{$\begin{array}{c}\text { Potencia [Hp] } \\
\text { Productividad } \\
\text { [kg/h] } \\
\text { rpm }\end{array}$} & $\begin{array}{l}\text { Modelo } \\
\text { UTC }\end{array}$ & $\begin{array}{l}\text { Modelo } \\
\text { GX_85 }\end{array}$ & $\begin{array}{l}\text { Modelo } \\
\text { GX-100 }\end{array}$ & $\begin{array}{l}\text { Modelo } \\
\text { GX-105 }\end{array}$ & $\begin{array}{c}\text { Modelo } \\
\text { E-250 }\end{array}$ & $\begin{array}{l}\text { Modelo } \\
\text { GX-130 }\end{array}$ & $\begin{array}{l}\text { Modelo } \\
\text { E-1000 }\end{array}$ \\
\hline & 6 & 7 & 15 & 17 & 48 & 25 & 124 \\
\hline & 30 & 70 & 175 & 250 & 250 & 400 & 1000 \\
\hline & 105 & 50 & 42 & 38 & 37 & 32 & \\
\hline \multicolumn{8}{|c|}{ MÁQUINAS CON DOBLE EXPELLER } \\
\hline & & GN-86 & GN-95 & GN-110 & GN-130 & GN-150 & \\
\hline Potencia [Hp] & & 7 & 15 & 15 & 25 & 30 & \\
\hline $\begin{array}{l}\text { Productividad } \\
{[\mathrm{kg} / \mathrm{h}]}\end{array}$ & & 110 & 200 & 250 & 400 & 500 & \\
\hline rpm & & 42 & 42 & 38 & 37 & 32 & \\
\hline
\end{tabular}

Fuente: (GLOBAL EXTENT S.R.L., 2019)

\section{Parámetros geométricos del tornillo.}

En la tabla 5. Se puede determinar la humedad de la materia prima que en muestro caso es $30 \%$, la temperatura no debe sobrepasar los $90^{\circ} \mathrm{C}$ y las revoluciones por minuto del tornillo so deben exceder los $95 \mathrm{rpm}$ que equivale a 10rad/s, siendo el sistema optimo el expeller de corte bajo. 
Tabla 5. Características de operación de un expeller de tornillo simple

\begin{tabular}{lccc}
\hline \multicolumn{1}{c}{$\begin{array}{c}\text { Variable de } \\
\text { operación }\end{array}$} & $\begin{array}{l}\text { Expeller de } \\
\text { corte bajo }\end{array}$ & $\begin{array}{l}\text { Expeller de } \\
\text { corte medio }\end{array}$ & $\begin{array}{l}\text { Expeller de } \\
\text { corte alto }\end{array}$ \\
\hline $\begin{array}{l}\text { Humedad de la } \\
\text { materia prima (\%) }\end{array}$ & $25-35$ & $20-30$ & $12-20$ \\
$\begin{array}{l}\text { Temperatura máxima } \\
\text { del producto }\left({ }^{\circ} \mathrm{C}\right)\end{array}$ & $50-120$ & $125-175$ & $150-200$ \\
$\begin{array}{l}\text { Relación Lt/Dt } \\
\text { Relación de } \\
\text { compresión }\end{array}$ & $5-8$ & $10-20$ & $4-12$ \\
$\begin{array}{l}\text { Velocidad del tornillo } \\
\text { (rad/s) }\end{array}$ & $3-1,10$ & $2-3: 1$ & $3-5: 1$ \\
$\begin{array}{l}\text { Energía mecánica neta } \\
\text { de entrada }(\mathrm{kW}-\mathrm{h} / \mathrm{kg})\end{array}$ & $0,03-0,06$ & $0,04-0,08$ & $0,10-0,14$ \\
\hline
\end{tabular}

Fuente: (Kokini, Tang Ho, \& Dekker, 1992)

Los datos de la geometría del tornillo se presentan en la tabla 6, los cuales fueron determinados en función de la geometría típica del tornillo extrusor mostrado en la figura 4, aplicados y detallados en la figura 5 .

Tabla 6. Parámetros geométricos del tornillo

\begin{tabular}{lccc}
\hline \multicolumn{1}{c}{ Parámetro } & Símbolo & Valor & Unidades \\
\hline Diámetro del tornillo & $\mathrm{Dt}$ & 66 & {$[\mathrm{~mm}]$} \\
Holgura camisa - tornillo & $\mathrm{Hct}$ & 0,5 & {$[\mathrm{~mm}]$} \\
Altura del hilo del tornillo & $\mathrm{Ht}$ & 7 & {$[\mathrm{~mm}]$} \\
Ancho del hilo del tornillo & $\mathrm{bt}$ & 9 & {$[\mathrm{~mm}]$} \\
Paso del tornillo & $\mathrm{pt}$ & 22 & {$[\mathrm{~mm}]$} \\
Longitud del tornillo & $\mathrm{Lt}$ & 340 & {$[\mathrm{~mm}]$} \\
Prelación L/D & $\mathrm{Lt} / \mathrm{Dt}$ & 5,15 & \\
Radio medio del tornillo & $\mathrm{rm}$ & 29,5 & {$[\mathrm{~mm}]$} \\
$\begin{array}{l}\text { Ángulo de hélice del } \\
\text { tornillo }\end{array}$ & $\theta \mathrm{t}$ & 6,77 & $\circ$
\end{tabular}


Ancho del canal del tornillo

$\mathrm{Wt} \quad 15,5 \quad[\mathrm{~mm}]$

Fuente: Elaboración propia.

Figura 4. Geometría típica de un tornillo extrusor.

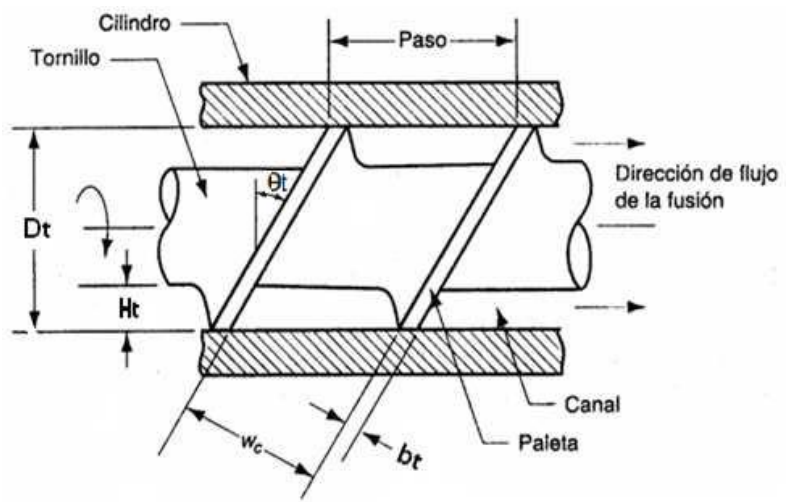

Fuente: (Mikell, 2007)

Figura 5. Tornillo sinfín.

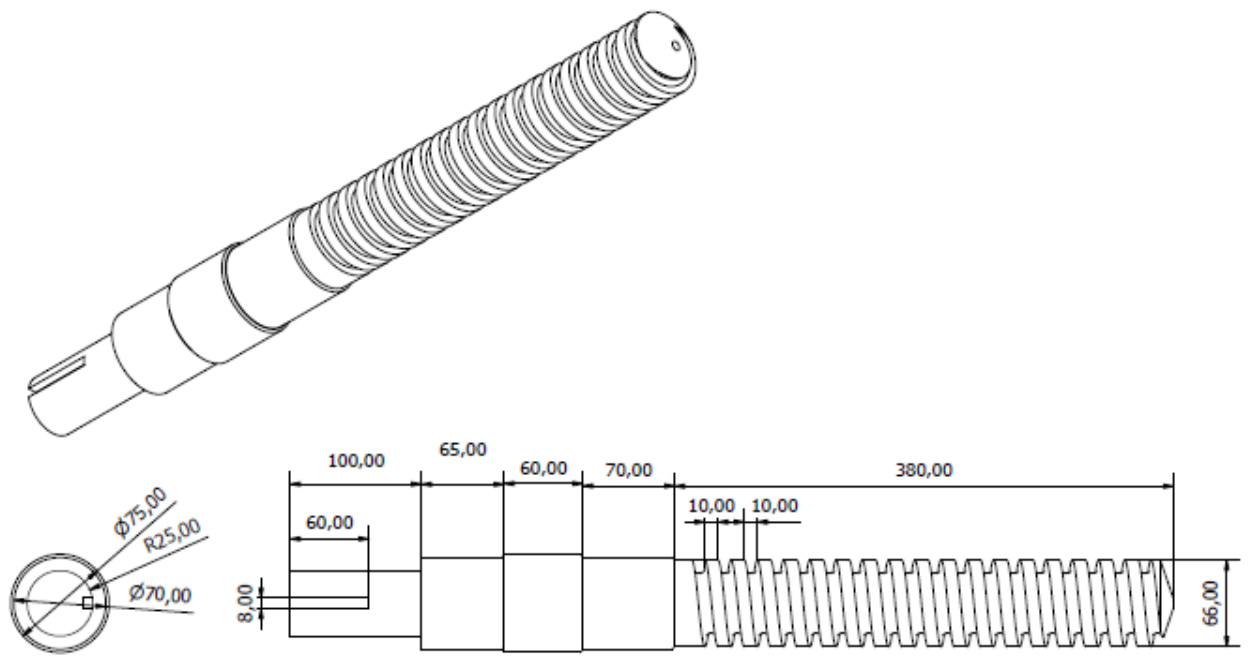

Fuente: Elaboración propia.

En la tabla 7 se resumen los motivos para la selección del acero inoxidable austenítico 304.

Tabla 7. Acero inoxidable austeníco serie 300.

\begin{tabular}{cccccc}
\hline Tipo de aleación & $\mathbf{C}$ & $\mathbf{S i}$ & $\mathbf{M n}$ & $\mathbf{C r}$ & $\mathbf{N i}$ \\
$\%$ & 0,03 & 0,5 & 1,4 & 18 & 8 \\
\hline Normas & AISI 304 & BOEHLER A604 & & & \\
Propiedades & Resistente a la corrosión, vapor de agua &
\end{tabular}


Aplicaciones

Equipo químico de procesos, manejo de alimentos y equipos para hospitales.

Fuente: (DIPAC, 2019)

\section{Cuerpos cilíndricos de la máquina}

Está constituido por el cuerpo extractor (figura 6), disco extractor de residuos, porta resistencias y el disco centrador. Estos elementos son construidos de acero bonificado AISI 4140. Las especificaciones se muestran en la tabla 8 .

Tabla 8. Características del material de los cuerpos cilíndricos de la máquina

\begin{tabular}{cccccc}
\hline Tipo de aleación & $\mathbf{C}$ & $\mathbf{S i}$ & $\mathbf{M n}$ & $\mathbf{C r}$ & $\mathbf{N i}$ \\
$\mathbf{\%}$ & 0,41 & 0,3 & 0,7 & 1,1 & 0,2 \\
\hline \multirow{2}{*}{ Normas } & AISI & BOEHLER V320 \\
Propiedades & 4140 & Resistencia a la fatiga y fractura. \\
Aplicaciones & \multicolumn{4}{c}{ Se utiliza en los cojinetes, cilindros, } \\
\multicolumn{4}{c}{ engranajes. } \\
\hline
\end{tabular}

Fuente: (ggdmetals, 2019)

Figura 6. Camisa de extracción

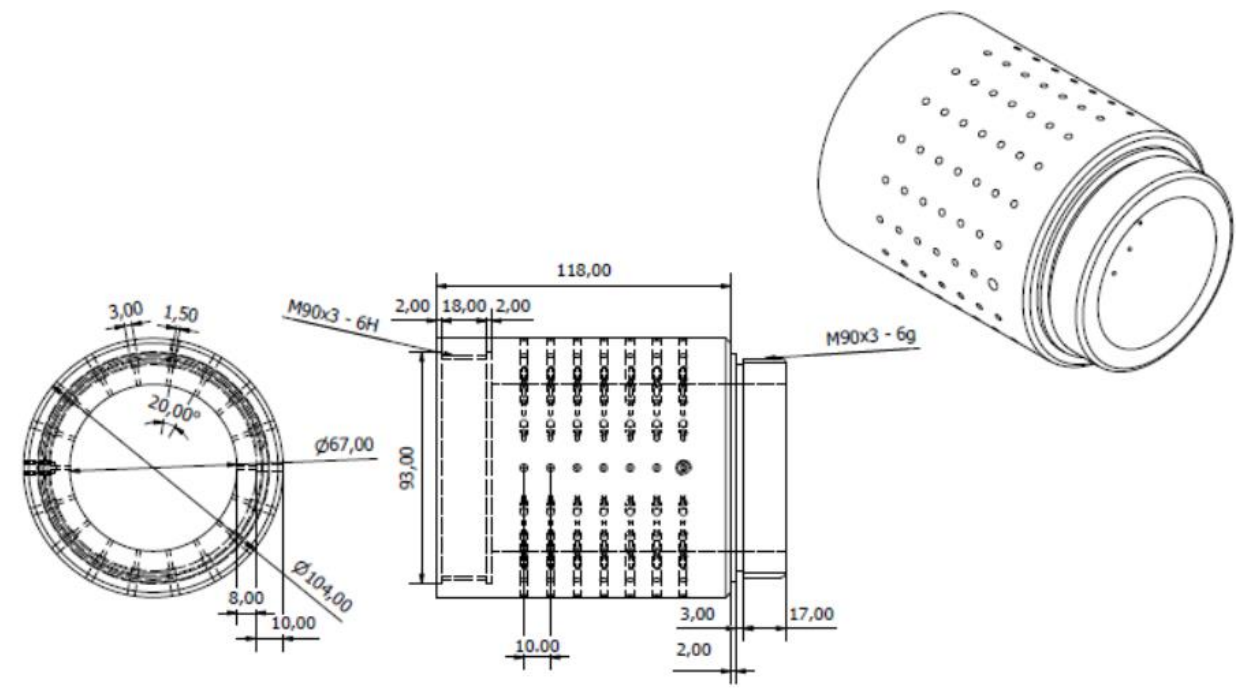

Fuente: Elaboración propia.

\section{Cuerpo principal y anillos de fijación}

En la figura 7 se presenta el alojamiento de los rodamientos, siendo el cuerpo principal debido a que contiene los rodamientos y es la central de la máquina. La tabla 9, presenta el tipo de acero que se utilizara para la construcción del mismo. 
Figura 7. Alojamiento de los rodamientos.
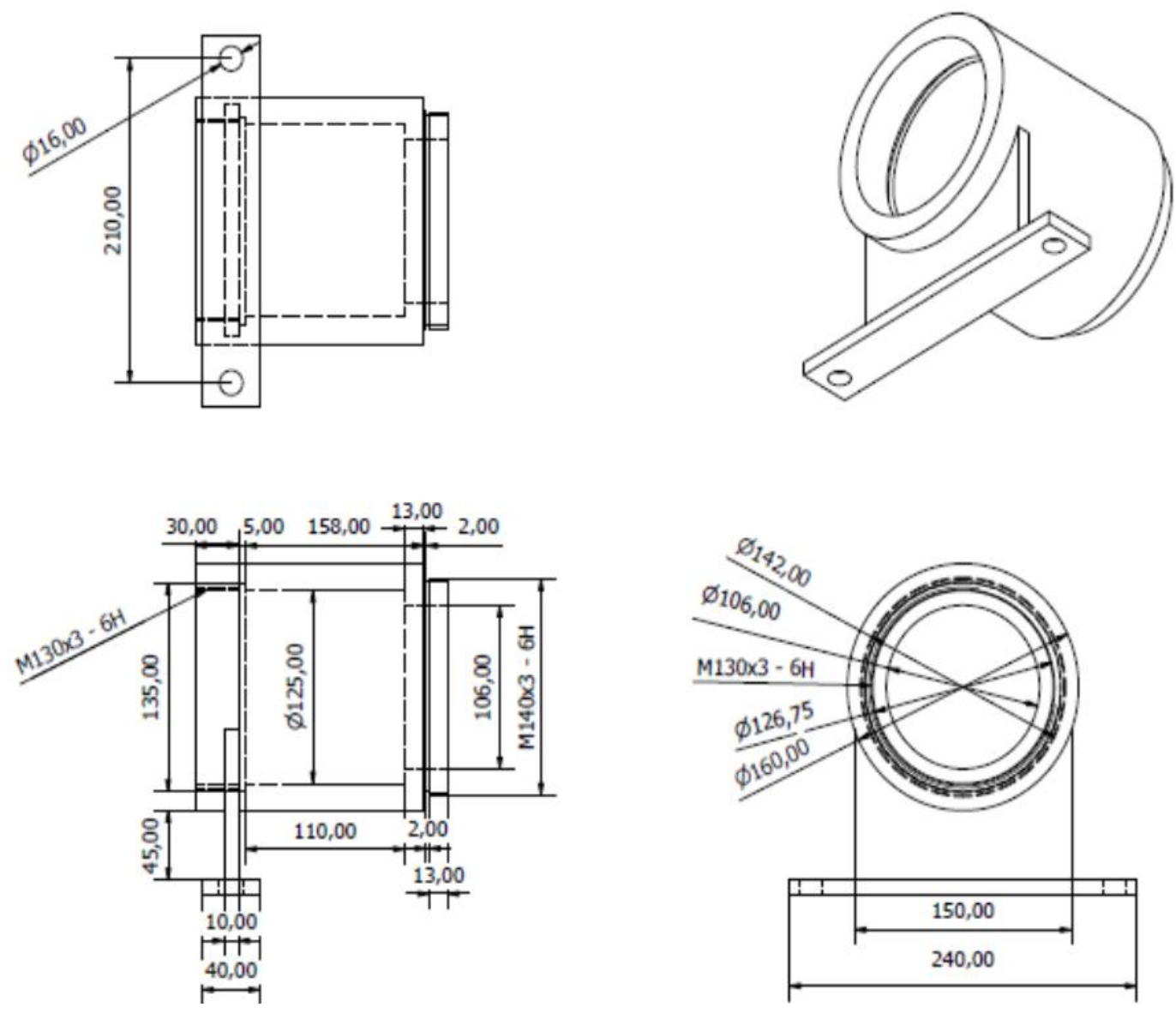

Fuente: Elaboración propia.

Tabla 9. Características del material de cuerpos cilíndricos de la máquina

\begin{tabular}{|c|c|c|c|c|c|c|}
\hline $\begin{array}{c}\text { Tipo de aleación } \\
\%\end{array}$ & $\begin{array}{c}\mathbf{C} \\
0,38\end{array}$ & $\begin{array}{r}\mathbf{S i} \\
0,3\end{array}$ & $\begin{array}{l}\text { Mn } \\
0,7\end{array}$ & $\begin{array}{l}\mathbf{C r} \\
1,1\end{array}$ & $\begin{array}{l}\text { Mo } \\
0,2\end{array}$ & $\begin{array}{l}\mathbf{N i} \\
1,5\end{array}$ \\
\hline & AISI & & & & & \\
\hline Normas & 4340 & BOEH & & & & \\
\hline Propiedades & resisten & la fatig & tura. & & & \\
\hline Aplicaciones & Se utiliz & las $m a$ & los ci & & & \\
\hline
\end{tabular}

Fuente: (ggdmetals, 2019)

\section{Selección de los rodamientos}

Los rodamientos seleccionados son cónicos porque a más de soportar el peso del conjunto de la máquina tienen que absorber la fuerza axial producto de la presión interna.

En base al programa SKF Bearing Select versión: 1.0.36. en línea se determinó el rodamiento 32214-J2-Q-SKF de las siguientes dimensiones: 70x125x33.3 mm, mostrado en la figura 8 . El rodamiento cónico seleccionado presenta una carga cinemática equivalente de $\mathrm{C}=125 \mathrm{kN}$ y una carga estática equivalente de $\mathrm{Co}=156 \mathrm{KN}$. 
Figura 8. Rodamiento cónico32214-J2-Q-SKF

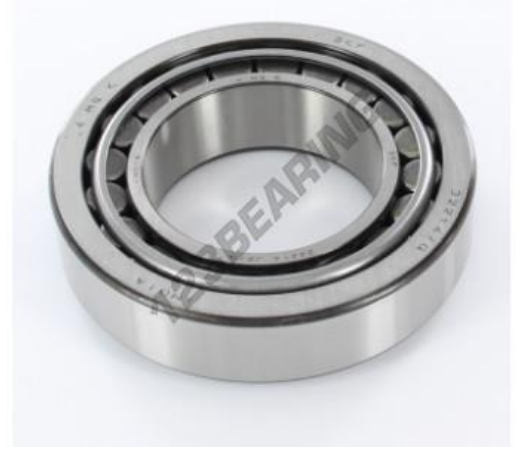

Fuente: (Rodamiento, 2019)

\section{Selección de los dispositivos eléctricos y electrónicos.}

Se calculó la corriente nominal de trabajo en función de la potencia del motor y se seleccionó los dispositivos de control y protección señalados en la tabla 10.

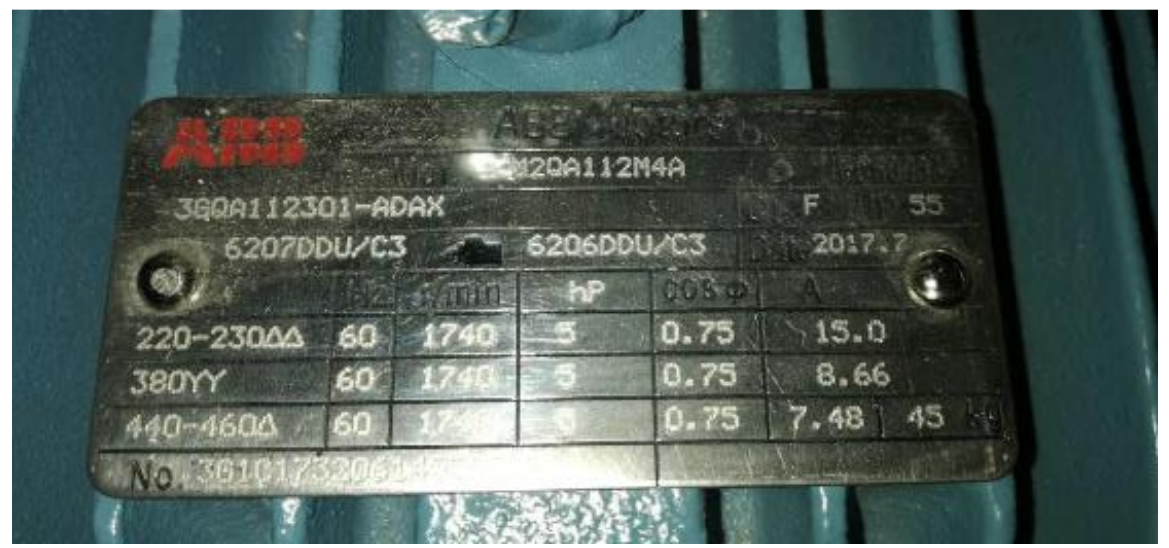

Fuente: Mercado Libre Ecuador.

Tabla 10. Variables para la selección de los equipos eléctricos

\begin{tabular}{lll}
\hline VARIABLE & VALOR & UNIDAD \\
\hline $\begin{array}{l}\text { Potencia motor } \\
\text { eléctrico }\end{array}$ & 4,8 & {$[\mathrm{~kW}]$} \\
$\begin{array}{l}\text { Voltaje disponible } \\
\text { Sistema Trifásico }\end{array}$ & 220 & {$[\mathrm{~V}]$} \\
$\begin{array}{l}\text { Factor de potencia } \\
\text { Corriente calculada }\end{array}$ & 0,75 & \\
$\begin{array}{l}\text { Constante para } \\
\text { selección de contactor }\end{array}$ & 16,80 & {$[\mathrm{~A}]$} \\
\end{tabular}


Corriente para

selección de contactor

Corriente para

selección del Breaker

Corriente para

selección de variador

Rango de temperatura

de operación
$20,15 \quad[\mathrm{~A}]$

$20,15 \quad[\mathrm{~A}]$

$16,80 \quad[\mathrm{~A}]$

$50-90 \quad\left[{ }^{\circ} \mathrm{C}\right]$

Fuente: Elaboración propia.

En base a las variables descritas en la tabla anterior, se seleccionan los dispositivos eléctricos y electrónicos para el sistema de control del motorreductor y del sistema de calentamiento de la máquina.

Tabla 11. Listado de equipos eléctricos y electrónicos

\begin{tabular}{lcc}
\hline \multicolumn{1}{c}{ ELEMENTO } & VALOR & UNIDAD \\
\hline Fusibles de 20 A gG & 20 & {$[\mathrm{~A}]$} \\
Contactor LC1DT20M7 & 20 & {$[\mathrm{~A}]$} \\
& 17 & {$[\mathrm{~A}]$} \\
$\begin{array}{l}\text { Variador ACS355-03E-17A6-2 } \\
\text { Resistencia tipo J 3/8x2 in }\end{array}$ & 100 & {$[\mathrm{~W}]$} \\
\hline
\end{tabular}

Fuente: Elaboración propia.

Figura 10. Portafusibles y fusibles de $20 \mathrm{~A}$ gG.
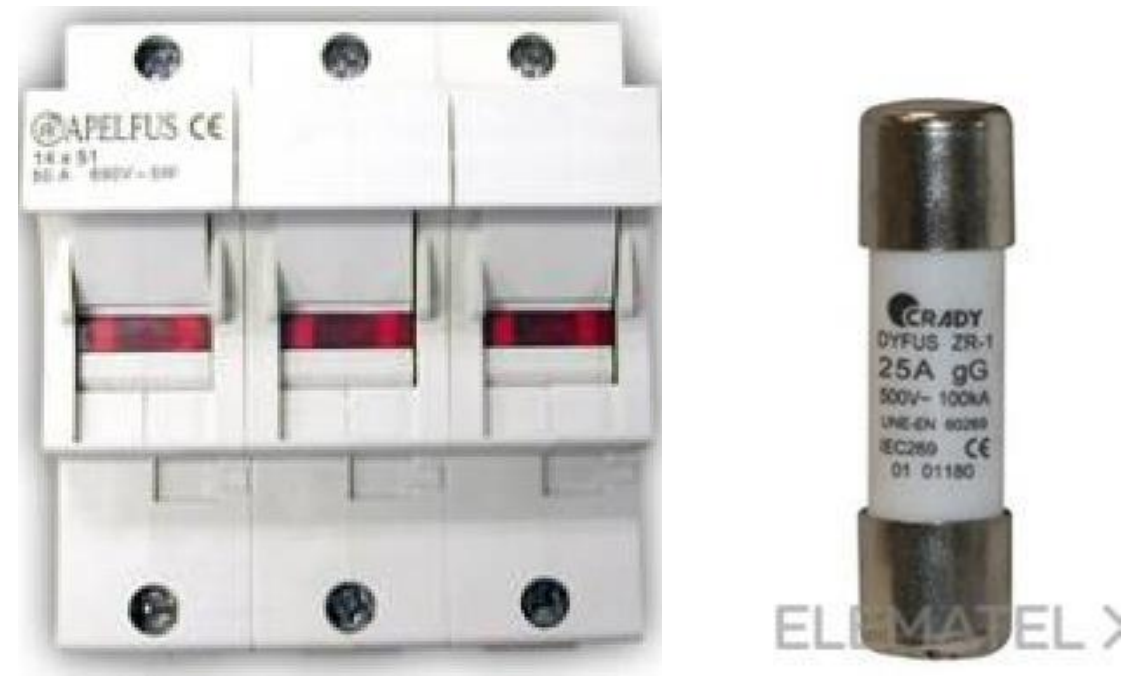

Fuente: Mercado Libre Ecuador. 
Figura 11. Contactor LC1DT20M7.

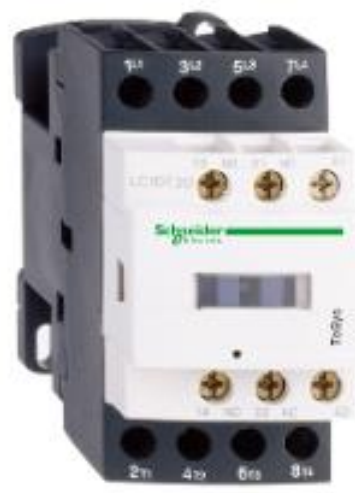

Fuente: Mercado Libre Ecuador.

Figura 12. Variador ACS355-03E-17A6-2.

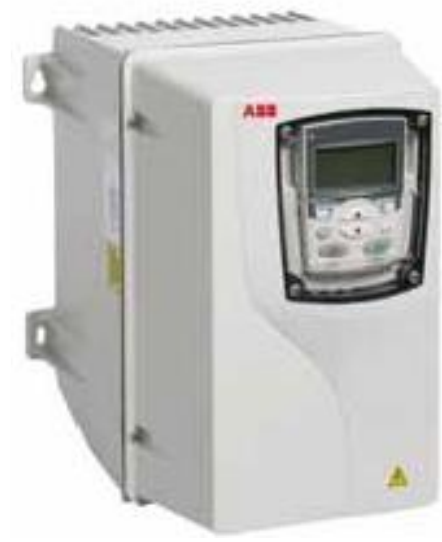

Fuente: Mercado libre Ecuador.

Figura 13. Resistencia tipo $\mathrm{J} 3 / 8 \times 2$ in.

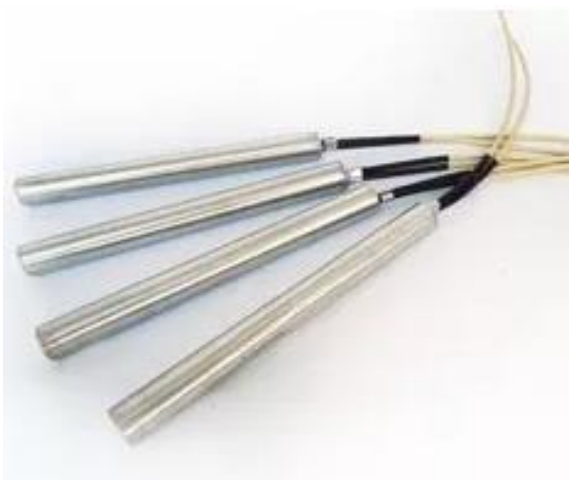

Fuente: Elaboración propia. 
Se seleccionó la termocupla tipo J puesto que su temperatura de operación varia de 0 a $750{ }^{\circ} \mathrm{C}$. Según el catálogo Resistencias de cartucho de la Empresa Kualitool.

Figura 14. Controlador de temperatura ATR2415.

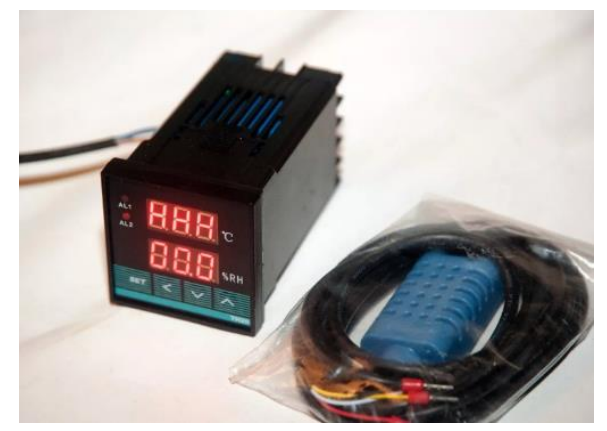

Fuente: Elaboración propia.

En las figuras 10,11,12,13,14; se presentan los elementos físicos que se emplearan para el sistema de fuerza y control de la máquina extrusora de tornillo.

La figura 15. Presenta el diagrama de potencia del motorreductor.

Figura 15. Diagrama de potencia del motorreductor

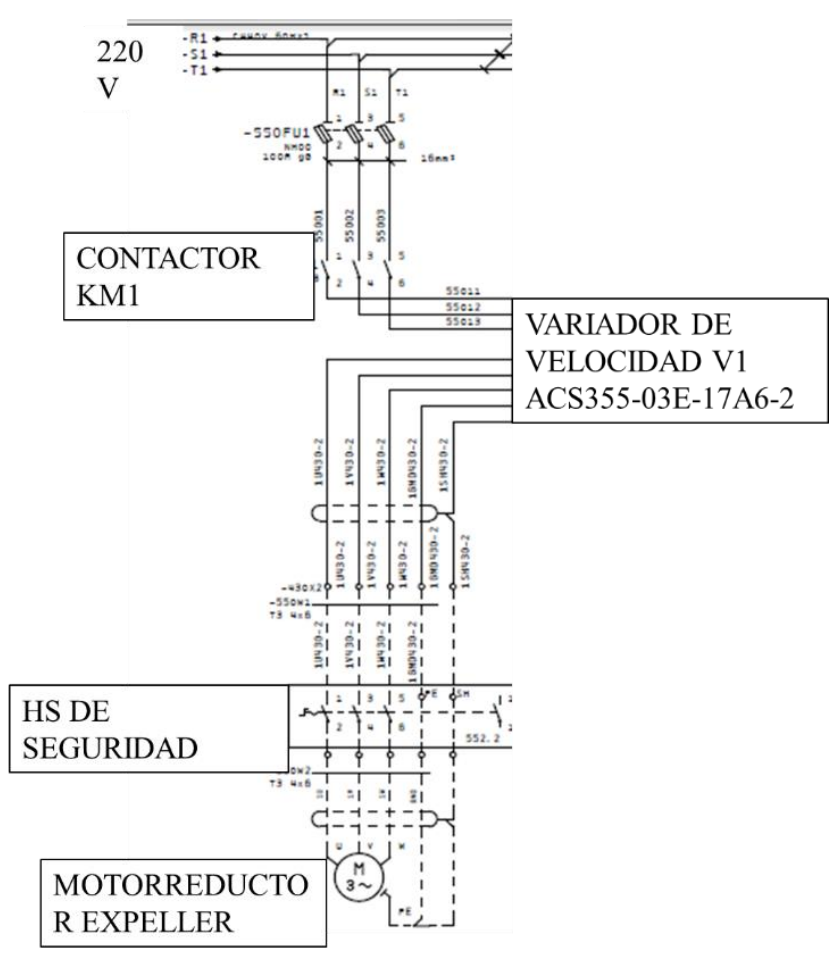

Fuente: Elaboración propia. 


\section{Conclusiones.}

- El diseño se logró obtener mediante el uso de todo el material bibliográfico poniendo especial atención a la potencia y revoluciones por minuto de las máquinas existentes en el mercado. En el proyecto se concluye que la máquina debe tener un motorreductor de $6 \mathrm{HP}$ y el tornillo debe girar a una revolución de $100 \mathrm{rpm}$.

- El proceso de extracción de aceite a través de la máquina expeller permitirá extraer mayor porcentaje de líquido de aceite del piñón ya que su operación es continua.

- De la comparación de máquinas disponible se concluye que la velocidad del tornillo influye de manera directa en la capacidad de producción, a mayor rpm existe menos producción de aceite, por ende, es fundamental no exceder los $100 \mathrm{rpm}$.

- Es fundamental poner atención a los catálogos tanto de materiales mecánicos y elementos eléctricos y electrónicos para no conllevar gastos innecesarios y también para alargar la vida útil de la máquina y no tener problemas de operación y funcionamiento.

- El proyecto una vez puesto en práctica, permitirá la enseñanza teórico-práctico durante la formación universitaria, porque los estudiantes conocerán el funcionamiento real de máquinas existentes en la industria de manufactura.

- Para la construcción de esta máquina de extracción de aceite se necesita invertir alrededor de 12000 USD, cabe mencionar que la diferencia de este tipo de maquina comparándola con las existentes en el mercado es la variación del rpm y el control de temperatura que permitirá la mayor eficiencia de la misma.

\section{Referencias Bibliográficas.}

Ali, S. (2016). Introduction to Bioplastics Engineering.

DIPAC. (2019). DIPAC PRODUCTOS DE ACERO. Manta, Ecuador. Obtenido de http://www.dipacmanta.com/acero-inoxidable-aisi

Evon, P., Kartika, A., \& Rigal, L. (2013). Extraction of oil from jatropha seeds using a twin-screw extruder: Feasibility study. Industrial Crops and Products, 33-42. doi:10.1016/j.indcrop.2013.02.034

ggdmetals. (2019). De las mejores industrias del mundo. Brazil. Obtenido de https://ggdmetals.com.br/es/produto/sae-4140/

GLOBAL EXTENT S.R.L. (2019). GLOBAL EXTENT S.R.L. Obtenido de https://www.gX.com.ar/gX-85

Global trade starts here. (2019). Alibaba.com. Obtenido de https://spanish.alibaba.com/productdetail/palm-oil-twin-screw-press-machine-60760616836.html

Gómez Vargas, S. J., \& Villegas Zapater, S. P. (14 de 01 de 2011). Diseño y construcción de una máquina tipo expeller para la extracción de aceite vegetal a partir de la semilla de la 
Jatropha Curcas con capacidad de 200 kilogramos/hora para la ESPE IASA II. Sangolquí, Pichincha.

Kokini, J., Tang Ho, C., \& Dekker, K. M. (1992). Food Extrusion Science and Technology. New York.

Mikell, G. (2007). Fundamentos de manufactura moderna. Prentice Hall.

MOTOVARIO. (2018). Motovario Catalogues. TECNICA INDUSTRIALE SRL. Estados Unidos.

Orisaleye, J., \& Ojolo, S. (2019). Parametric analysis and design of straight screw extruder for solids compaction. Journal of King Saud University - Engineering Sciences, 86-96. doi:10.1016/j.jksues.2017.03.004

Ramiréz, V., Martí, J., \& Rivadeneira, D. (2019). Energy use of Jatropha oil extraction wastes: Pellets from biochar and Jatropha shell blends. Journal of Cleaner Production, 1095-1102. doi:10.1016/j.jclepro.2019.01.132

Rodamiento, 1. (2019). 123 Rodamiento. Ecuador. Obtenido de https://www.123rodamiento.es/rodamiento-32214-J2-Q-SKF.php

Uitterhaegen, E., \& Evon, P. (2017). Tecnología de extrusión de doble tornillo para la extracción de aceite vegetal: una revisión. Revista de Ingeniería de Alimentos, 190-200. doi:10.1016/j.jfoodeng.2017.06.006.

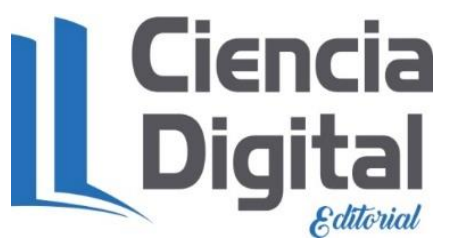


PARA CITAR EL ARTÍCULO INDEXADO.

Vásquez Carrera, P. J., Hidalgo Osorio, W. A., Carrillo Velarde, G. G., \& Villarroel Herrera, N. J. (2020). Selección y Diseño de una Máquina extractora de aceite del piñón Jatropha curcas. ConcienciaDigital, 3(4), 26-44. https://doi.org/10.33262/concienciadigital.v3i4.1423

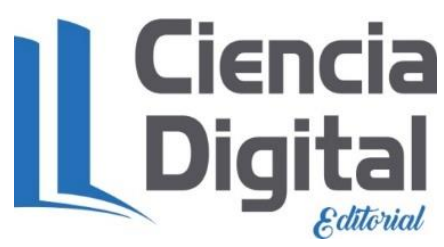

El artículo que se publica es de exclusiva responsabilidad de los autores y no necesariamente reflejan el pensamiento de la Revista Conciencia Digital.

El artículo queda en propiedad de la revista y, por tanto, su publicación parcial y/o total en otro medio tiene que ser autorizado por el director de la Revista Conciencia Digital.
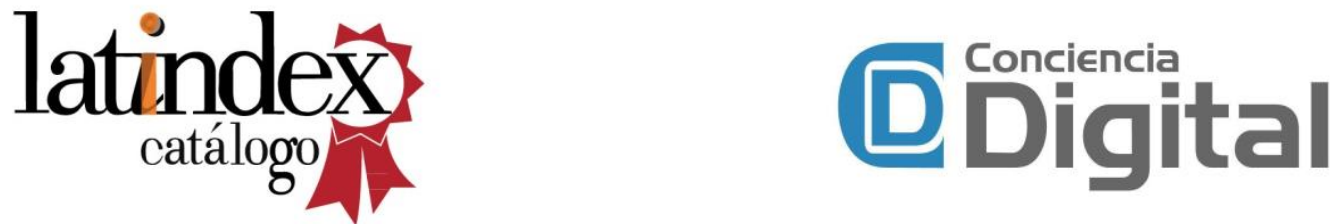